\title{
Nitric oxide affects salt-induced changes in free amino acid levels in maize
}

Ákos Boldizsár, Livia Simon-Sarkadi, Krisztina Szirtes, Alexandra Soltész, Gabriella Szalai, Marshall Keyster, Ndiko Ludidi, Gábor Galiba and Gábor Kocsy

\begin{abstract}
:
It was assumed that salt-induced redox changes affect amino acid metabolism in maize (Zea mays L.), and this influence may be modified by NO. The applied $\mathrm{NaCl}$ treatment reduced the fresh weight of shoots and roots. This decrease was smaller after the combined application of $\mathrm{NaCl}$ and an $\mathrm{NO}$-donor ((Z)-1[N-(2-aminoethyl)-N-(2-ammonioethyl)amino]diazen-1-ium-1,2-diolate, $E T A / N O$ ) in the shoots, while it was greater after simultaneous treatment with $\mathrm{NaCl}$ and nitro-l-arginine (l-NNA, inhibitor of NO synthesis) in the roots. The quantum yield efficiency of photosystem II was not influenced by the treatments. $\mathrm{NaCl}$ had a significant effect on the redox environment in the leaves as it was shown by the increase in the amount of glutathione disulphide and in the redox potential of the glutathione/glutathione disulphide redox pair. This influence of $\mathrm{NaCl}$ was modified by DETA/NO and lNNA. Pharmacological modification of NO levels affected salt-induced changes in both the total free amino acid content and in the free amino acid composition. $\mathrm{NaCl}$ alone increased the concentration of almost all amino acids which effect was strengthened by DETA/NO in the case of Pro. 1-NNA treatment resulted in a significant increase in the Ala, Val, Gly and Tyr contents. The Ile, Lys and Val concentrations rose considerably after the combined application of $\mathrm{NaCl}$ and DETA/NO compared to $\mathrm{NaCl}$ treatment alone in the recovery phase. $\mathrm{NaCl}$ also increased the expression of several genes related to the amino acid and antioxidant metabolism, and this effect was modified by DETA/NO. In conclusion, modification of NO levels affected salt-induced, glutathione-dependent redox changes and simultaneously the free amino acid composition and the level of several free amino acids. The observed much higher Pro content in plants treated with both $\mathrm{NaCl}$ and DETA/NO during recovery may contribute to the protective effect of NO against salt stress.
\end{abstract}

\section{Introduction}

Adverse environmental conditions result in substantial reductions in crop yields. Yield losses caused by drought can be mitigated by irrigation, but this may lead to the deposition of salt from the river water or groundwater applied. High salt 
concentrations induce oxidative stress due to an increase in the amount of reactive oxygen species, so antioxidants are activated as a part of the defence system (Foyer and Noctor, 2011). The accumulation of amino acids, especially Pro and Arg, contributes to the osmoprotection of plants (Rai, 2002). Increase in Arg content may lead to the accumulation of polyamines which protect as polycations the vital negatively charged, macromolecules in the cells (Alcázar et al., 2006). In addition, reactive oxygen species, antioxidants, amino acids and polyamines are all involved in signalling pathways which may activate further protective mechanisms. During salt stress there is cross-talk between various signalling pathways involving reactive oxygen, nitrogen species and plant growth regulators (Moreau et al., 2010; Gémes et al., 2011).

The involvement of antioxidants in the response to salt stress was shown in maize, where $\mathrm{NaCl}$ treatment increased both the catalase and superoxide dismutase (SOD) transcript levels and the catalase activity (Menezes-Benavente et al., 2004). Transcriptome analysis revealed a rapid induction of genes encoding antioxidants by salt in a tolerant poplar species, while their induction occurred only after longer exposure to salt in the sensitive one (Ding et al., 2010). Salt stress increased the activity of SOD, ascorbate peroxidase (APX) and catalase in chickpea leaves (Sheokand et al., 2008). Further evidence for the protective role of antioxidants was observed in rice, since a salt-tolerant genotype was found to have greater ascorbate and glutathione (GSH) contents, GSH/glutathione-disulphide (GSSG) and ascorbate/dehydroascorbate ratios and antioxidant enzyme activity than a sensitive one (Vaidyanathan et al., 2003; ElShabrawi et al., 2010). Similarly, the salt-tolerant species Plantago maritima exhibited better tolerance to salt stress than the salt-sensitive Plantago media due to its greater antioxidant activities (APX, glutathione reductase (GR), SOD, catalase) (Sekmen et al., 2007). The salinity tolerance of Medicago trunculata was found to be related to the induction and sustained expression of highly regulated antioxidant mechanisms in the roots and leaves (Mhadhbi et al., 2011). In the induction of antioxidants the osmotic effect of salt may be important, since osmotic stress induced by the water deficit also affected antioxidant levels (Varga et al., 2012). Besides antioxidants, amino acids are also important in the response to salt stress. In broad bean the amino acid content was decreased by increasing salinity and a marked increase was only observed in the proline content (Abd El-Samad et al., 2011). Although Wang et al. (2003) found no alteration in the overall free amino acid concentration in maize, the amino acid composition was changed by salt stress, with an increase in the proline and asparagine contents. Salt-induced changes in amino acid levels affect polyamine concentrations as it was shown in wheat (Simon-Sarkadi et al., 2007). All these findings indicate that the level of antioxidants, amino acids and polyamines are adjusted to salt stress conditions, which may improve salt tolerance.

NO was found to serve as a signal inducing salt tolerance in reed (Zhao et al., 2004). NO enhanced salt tolerance in maize due to the activation of proton pumps and the $\mathrm{Na}^{+} / \mathrm{H}^{+}$antiport, as indicated by improved growth, increased dry matter accumulation and greater chlorophyll content (Zhang et al., 2006). The protective 
effect of NO against salt stress in maize can also be linked to the enhanced activity of antioxidant enzymes (Bai et al., 2011; Keyster et al., 2012). The induction of various antioxidant enzymes (catalase, APX, SOD) by NO was also observed under salt stress in chickpea (Sheokand et al., 2008), wheat (Zheng et al., 2009) and rice (Uchida et al., 2002). The possible effect of NO on amino acids during salt stress was only investigated in the case of Pro in cucumber where the NO-induced increase in Pro was assumed to be responsible for an improved salt tolerance (Fan et al., 2012).

According to our hypothesis salt-induced redox changes may affect amino acid metabolism in maize and in this process NO may be involved, too. To test this assumption, free amino acid concentrations were measured in plants subjected to salt stress without and with simultaneous modification of NO levels and possible redox changes were monitored by determination of GSH and GSSG concentrations.

\section{Materials and methods}

\section{Plant material and growth conditions}

Maize (Zea mays L. cv. Silverking) seeds were imbibed in sterile distilled water for 30 min and sown in $1 \mathrm{l}$ of pre-soaked (distilled water) filtered silica sand in $15 \mathrm{~cm}$ diameter plastic pots. The seedlings were grown in a PGR-15 growth chamber (Conviron, Canada) at $22{ }^{\circ} \mathrm{C}, 400 \mu \mathrm{mol} \mathrm{m}-2 \mathrm{~s}^{-1}$ and $12 \mathrm{~h}$ illumination, and irrigated with modified Hoagland solution (Kello" s et al., 2008) twice a week. The following treatments were applied as supplementation of the nutrient solution at the 3-leaf stage of the plants: (1) control (addition of no chemical); (2) $150 \mathrm{mM} \mathrm{NaCl}$; (3) $5 \mu \mathrm{M}$ (Z)-1-[N-(2-aminoethyl)-N-(2ammonioethyl)amino]diazen-1-ium-1,2-diolate (DETA/NO); (4) $5 \mu \mathrm{M} D E T A / N O$ + $150 \mathrm{mM} \mathrm{NaCl}$; (5) $5 \mu \mathrm{M}$ diethylenetriamine (DETA); (6) $5 \mu \mathrm{M}$ DETA + $150 \mathrm{mM}$ $\mathrm{NaCl}$; (7) $5 \mu \mathrm{M}$ nitro-l-arginine (l-NNA); (8) $5 \mu \mathrm{M}$ l-NNA + $150 \mathrm{mM} \mathrm{NaCl}$; (9) 150 $\mathrm{mM} \mathrm{KNO} 2$. DETA/NO is a nitric oxide donor. DETA, which is produced by the dissociation of DETA/NO, was included among the treatments to act as a control for the NO treatments. Interestingly, it was found to affect the As-induced oxidative stress in maize (Stoeva et al., 2005). l-NNA is an inhibitor of NO synthesis, while $\mathrm{KNO}_{2}$ is a possible endogenous source of NO. The treatments were followed by a 1-week recovery phase when no chemicals were added to the basic nutrient solution. Sampling and the determination of fresh weight were done before the addition of the various compounds, after 3 and 11 days treatment and after 1 week recovery. Three independent experiments were performed with three-three parallels.

\section{Measurement of the quantum yield efficiency of photosystem II}

The maximum potential quantum efficiency of photosystem II was characterized by the $F_{\mathrm{V}} / F_{\mathrm{m}}$ (variable to maximum fluorescence) ratio which was measured using a pulse amplitude-modulated fluorometer (PAM 2000, Walz, Germany). 


\section{Analysis of thiols}

The qualitative and quantitative identification of the thiols was performed using reverse-phase HPLC (Waters, Milford, MA, USA) connected to a fluorescence detector (W474 scanning fluorescence detector, Waters) as previously described (Kranner and Grill, 1996; Kocsy et al., 2001). The half-cell reduction potential of the thiol/thiol disulphide redox couples was calculated according to Schafer and Buettner (2001).

\section{Determination offree amino acid content}

Shoot samples of 300-600 mg fresh weight were crushed in liquid nitrogen and extracted with $2 \mathrm{ml}$ cold $10 \%$ trichloroacetic acid for $1 \mathrm{~h}$ with gentle agitation on a shaker (C. Gerhardt GmbH \& Co. KG, Germany) at room temperature. Each sample was filtered through a $0.2 \mu \mathrm{m}$ pore membrane filter (Sartorius AG, Germany). The biochemical analysis was carried out on an automatic amino acid analyser (Ingos Ltd., Czech Republic) equipped with an Ionex Ostion LCP5O2O cation-exchange column $(22 \mathrm{~cm} \times 0.37 \mathrm{~cm})$. The free amino acids were separated by stepwise gradient elution using a $\mathrm{Li}^{+}$-citric buffer system (Ingos Ltd., Czech Republic). Colorimetric detection was accomplished at $570 \mathrm{~nm}$ and $440 \mathrm{~nm}$ (for Pro) after post-column derivatization with ninhydrin reagent.

\section{Gene expression studies}

For the real-time PCR analysis of the genes encoding enzymes involved in the amino acid and polyamine metabolism or in the antioxidant defence and death processes, the DNase treatment of RNA and first-strand cDNA synthesis were carried out according to Altpeter et al. (2005). The PCR amplification mixture (20 $\mu \mathrm{l}$ ) contained $0.5 \mu \mathrm{l} \mathrm{cDNA}, 10 \mu \mathrm{l}$ QuantiTect SYBR Green PCR Master mix (Qiagen, Hilden, Germany) and $0.8 \mu$ forward and reverse primer $(10 \mu \mathrm{M})$. The primers are listed in Supplementary Table S1. The amplification of DNA and normalization against glyceraldehyde-3-phosphate dehydrogenase (Kocsy et al., 2010) were performed as described earlier (Altpeter et al., 2005).

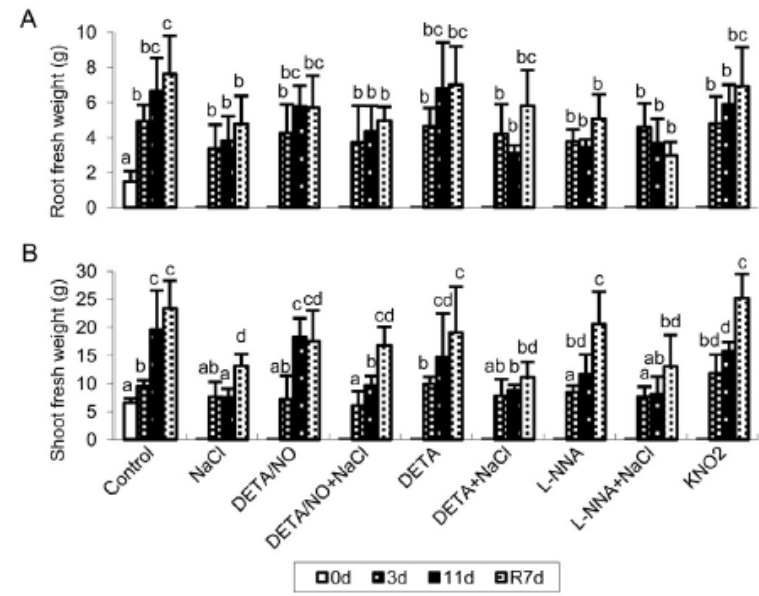

Fig. 1. Effect of $\mathrm{NO}$ on salt-induced changes in fres $h$ weight. The fresh weight of roots (A; significant difference, SD: 0.46 ) and shoots (B; SD: 0.69 ) was measured after 0 , 3 and 11 days of treatment with various compounds and after 7 days of recovery (without these compounds). NaCl: $150 \mathrm{mM}$; DETA/NO (NO-donor): $5 \mu \mathrm{mol}$; DETA: $5 \mu \mathrm{mol}$; L-NNA (inhibitor of NO-synthesis): $1 \mathrm{mM}$; $\mathrm{KNO}_{2}$ (precursor of $\mathrm{NO}$ ): $150 \mathrm{mM}$. The values indicated by different letters are significantly different at $p \leq 0.05$ level. 

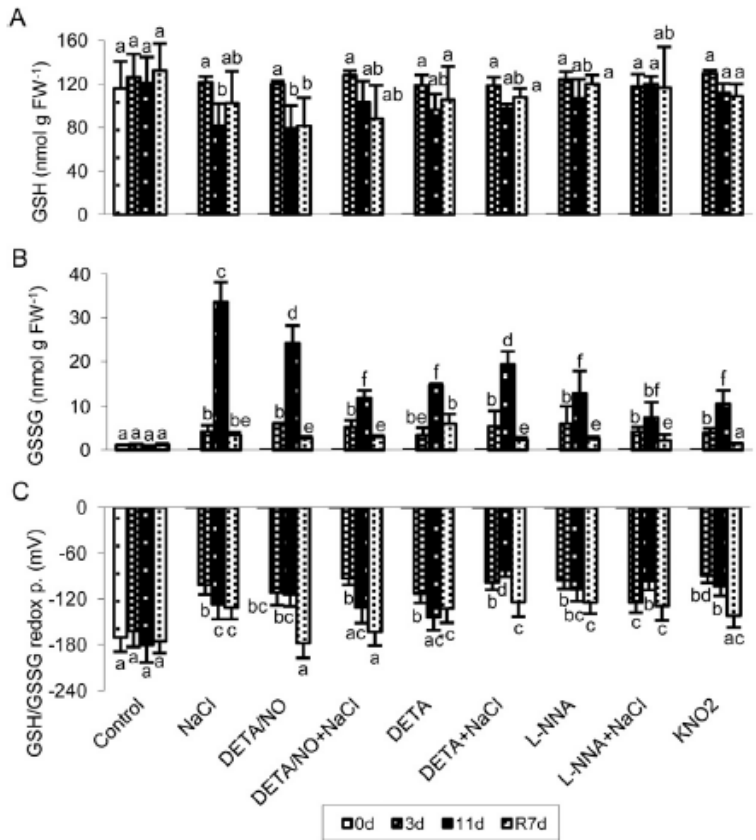

Fig. 2. Effect of NO on salt-induced changes in glutathione and glutathione disulphide content and in their redox potential. The amounts of glutathione (GSH, A; SD: 13.1) and glutathione disulphide (GSSG, B; SD: 1.2) and the redox potential of the GSH/GSSG redox couple (C; SD: 19.3 ) were measured in the youngest fully developed leaves after 0,3 and 11 days of treatment with various compounds and after 7 days of recovery (without these compounds). NaCl: $150 \mathrm{mM}$; DETA/NO (NO-donor): $5 \mu \mathrm{mol}$; DETA: $5 \mu \mathrm{mol}$; L-NNA (inhibitor of NO-synthesis): $1 \mathrm{mM}$; $\mathrm{KNO}_{2}$ (precursor of NO): $150 \mathrm{mM}$. The values indicated by different letters are significantly different at $p \leq 0.05$ level.

\section{Statistics}

The statistical analysis was done using two-component (treatments, samplings) analysis of variance. Significant differences (SD) were calculated with the $t$-test at the $p \leq 0.05$ level.

\section{Results}

\section{Fresh weight}

$\mathrm{NaCl}$ reduced the fresh weight of both roots (to 63\%) and shoots (to 38\%) compared to the control plants, but the difference was greater for the shoots after 11 days (Fig. 1). The inhibition of NO synthesis in salt-stressed maize resulted in an additional reduction in root growth to $58 \%$ during the recovery phase compared to plants treated only with $\mathrm{NaCl}$ (Fig. 1A). DETA/NO, added simultaneously with $\mathrm{NaCl}$ was able to restore to $74 \%$ of control the salt-induced growth inhibition of the shoots (Fig. 1B). Like the NO donor DETA/NO, the addition of $\mathrm{KNO}_{2}$, a possible source of NO in plants, did not affect the growth of the plants.

\section{Quantum yield efficiency of photosystem II}

The quantum yield efficiency of photosystem II decreased slightly in the course of the experiment but the various compounds did not induce significant changes in this parameter (Supplementary Fig. S1).

Supplementary material related to this article found, in the online version, at http://dx.doi.org/10.1016/j.jplph.2013.02.006. 


\section{Thiols, thiol disulphides and their redox potentials}

With the exception of $\mathrm{NaCl}$ and l-NNA $+\mathrm{NaCl}$ all the compounds induced a great reduction (to 26-40\%) in the cysteine content (precursor of GSH) during the recovery phase, and some of the treatments (DETA $+\mathrm{NaCl}$, l-NNA $+\mathrm{NaCl}, \mathrm{KNO}_{2}$ ) increased the cystine content (to $316 \%, 202 \%$ and $267 \%$ ) after 11 days salt stress

(Supplementary Figs. S2A and S2B). The treatments increased the redox potential of the cysteine/cystine couple (by $24-48 \%$, end of recovery), except for l-NNA + $\mathrm{NaCl}$ (Supplementary Fig. S2C).

Similarly to cysteine, the greatest changes in the -y-glutamylcysteine (-yEC) content (intermediary product of GSH synthesis) occurred during the recovery phase (Supplementary Fig. S3A), when its level was greatly reduced by DETA/NO (to $44 \%$ ), DETA + $\mathrm{NaCl}$ (to 42\%), l-NNA (to $33 \%$ ) and $\mathrm{KNO}_{2}$ (to 28\%). While the $-\mathrm{y}-$ glutamylcystine (ESSE) content was not affected by $\mathrm{NaCl}$ alone, its concentration was increased by the other treatments (to $127-248 \%$, end of recovery) with the exception of DETA/NO + NaCl (Supplementary Fig. S3B). The redox potential of the EC/ESSE redox pair was not affected by salt, but was increased by the addition of DETA/NO (by 25\%), l-NNA (by 30\%) and $\mathrm{KNO}_{2}$ (by 36\%) during the recovery (Supplementary Fig. S3C).

The amount of GSH exhibited only slight changes after the addition of the various compounds for 11 days except for its decrease after $\mathrm{NaCl}$ (to 67\%) and DETA/NO (to $65 \%$ ) treatment (Fig. 2A), but all the treatments resulted in a great increase (8-37fold) in the GSSG concentration after 11 days (Fig. 2B). The greatest changes were induced by $\mathrm{NaCl}$ (37-fold) and DETA/NO (27-fold). Both DETA/NO and l-NNA greatly reduced (to $35 \%$ and $22 \%$ ) the NaCl-induced increase in GSSG content. The redox potential of the GSH/GSSG couple increased (by 50-55\%, 11 days) after the treatments, and it remained at high level during the recovery phase except for DETA/NO and DETA/NO $+\mathrm{NaCl}$ treatments (Fig. 2C).

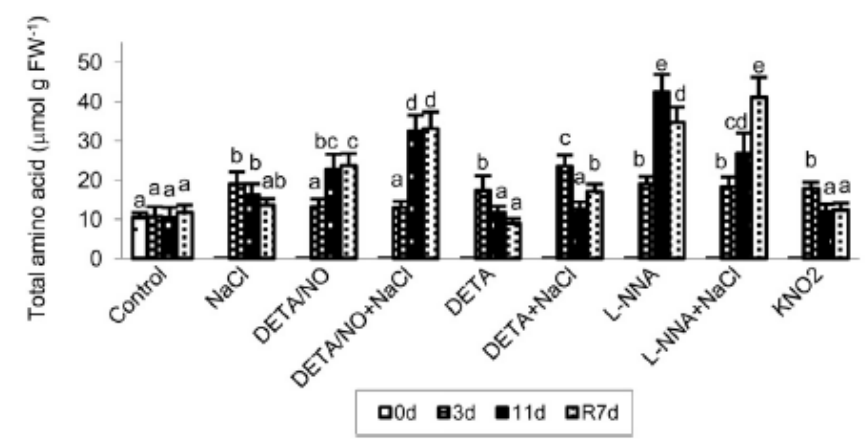

Fig. 3. Effect of NO on total free amino acid content. The free amino acid concentrations were measured in the youngest fully developed leaves after 0,3 and 11 days of treatment with various compounds and after 7 days of recovery (without these compounds). NaCl: $150 \mathrm{mM}$; DETA/NO (NO-donor): $5 \mu \mathrm{mol}$; DETA: $5 \mu \mathrm{mol}$; L-NNA (inhibitor of NO-synthesis): $1 \mathrm{mM} ; \mathrm{KNO}_{2}$ (precursor of $\mathrm{NO}$ ): $150 \mathrm{mM}$. The values indicated by different letters are significantly different at $p \leq 0.05$ level. SD: 3.4. 


\section{Free amino acids}

All treatments increased the total free amino content at least in one sampling point (Fig. 3). However, the greatest increase was observed after the addition of DETA/NO + $\mathrm{NaCl}$ (to 218\%), l-NNA (to 408\%) and l-NNA + $\mathrm{NaCl}$ (to 257\%) for 11 days. Not only the total free amino acid content, but the amino acid composition was affected by the treatments, too (Fig. 4). The ratio of the amino acids belonging to the aspartate family decreased (by $17 \%$ and $15 \%$ ) and that of the amino acids of glutamate and alanine family increased (by $7 \%$ and $5 \%$, by $10 \%$ and $7 \%$ ) after 3 days treatment with DETA/NO and DETA/NO + NaCl. After 11 days DETA/NO partly neutralized the effect of $\mathrm{NaCl}$, and the ratios after the application of DETA/ $\mathrm{NO}+\mathrm{NaCl}$ became more similar (1-7\% difference) to control than after the addition of $\mathrm{NaCl}$ (12-15\% difference) alone.

A

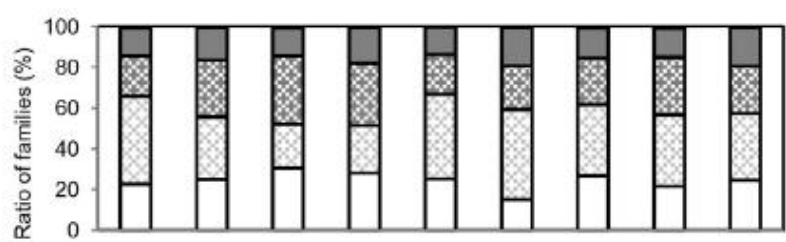

B

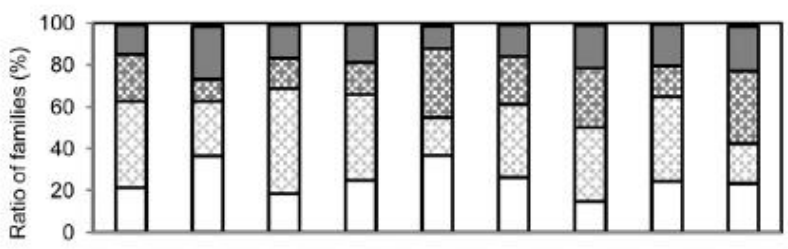

C

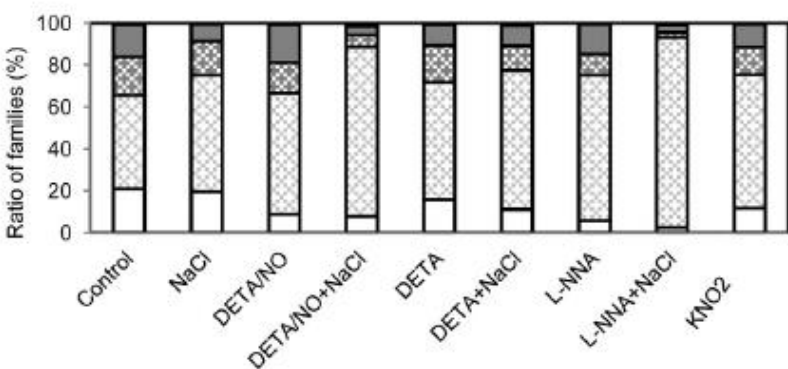

QGlutamte family

口Serine family

DAspartate family

- Aromatic amino acid family

DAlanine family

Fig. 4. Effect of $\mathrm{NO}$ on the ratio of amino acids belonging to the different amino acid families. The ratio was determined in the youngest fully developed leaves after 0 , 3 and 11 days of treatment with various compounds and after 7 days of recovery (without these compounds). NaCl: $150 \mathrm{mM}$; DETA/NO (NO-donor): $5 \mu \mathrm{mol}$; DETA: $5 \mu$ mol; L-NNA (inhibitor of NO-synthesis): $1 \mathrm{mM} ; \mathrm{KNO}_{2}$ (precursor of NO): $150 \mathrm{mM}$. The ratio of amino acids belonging to the aromatic amino acid family was between 0.4 and 1.6. SD: 2.3 

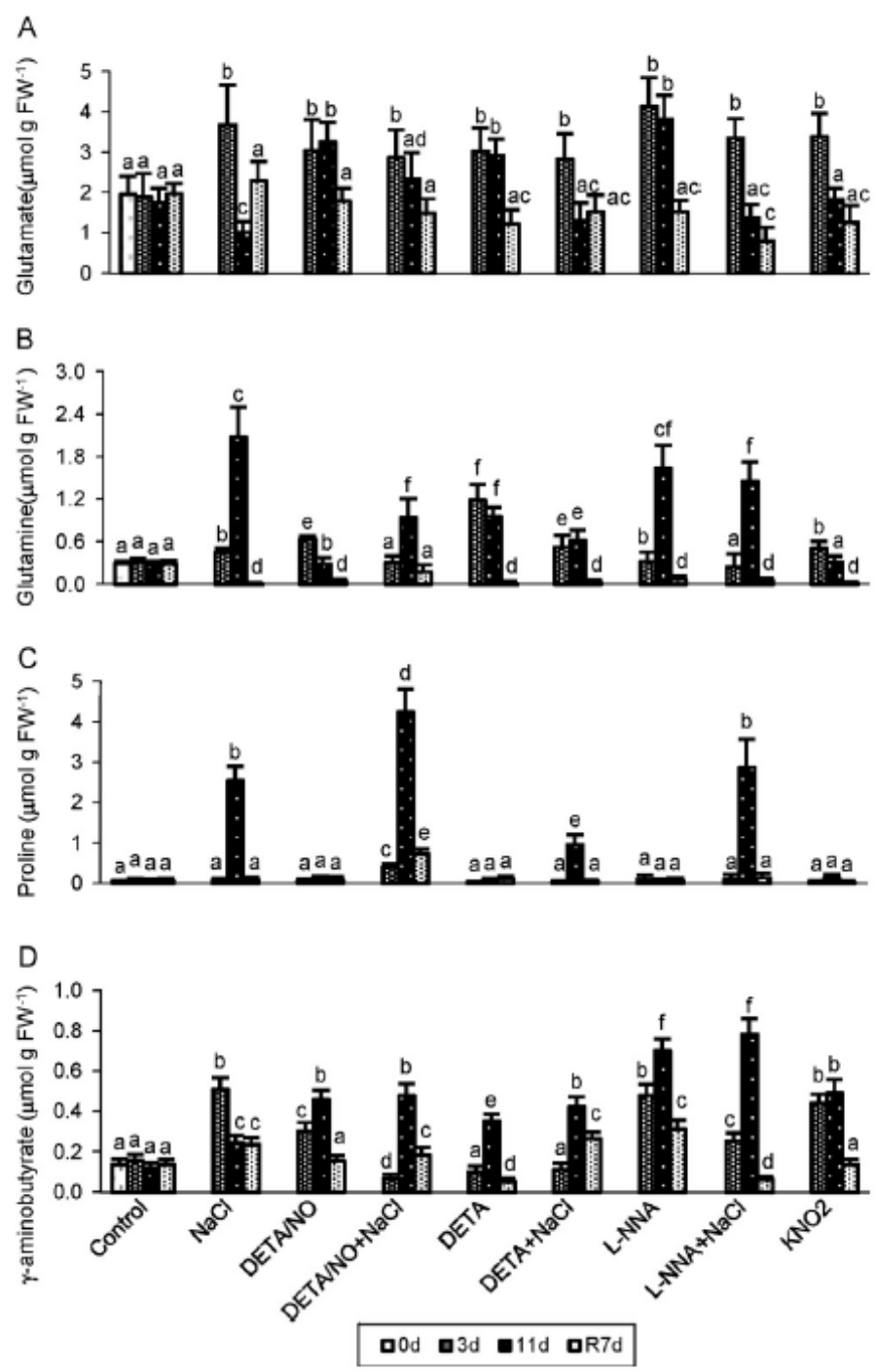

Fig. 5. Effect of NO on salt-induced changes in the concentration of amino acids belonging to the glutamate family. The amounts of glutamate (A; SD: 0.25 ), glutamine (B; SD: 0.063), proline (C; SD: 0.92) and $\gamma$-aminobutyrate (D; SD: 0.021 ) were measured in the youngest fully developed leaves after 0,3 and 11 days of treatment with various compounds and after 7 days of recovery (without these compounds). $\mathrm{NaCl}: 150 \mathrm{mM}$; DETA/NO (NO-donor): $5 \mu \mathrm{mol}$; DETA: $5 \mu \mathrm{mol}$; L-NNA (inhibitor of NO-synthesis): $1 \mathrm{mM} ; \mathrm{KNO}_{2}$ (precursor of $\mathrm{NO}$ ): $150 \mathrm{mM}$. The values indicated by different letters are significantly different at $p \leq 0.05$ level.

Interestingly, at the end of the 7 days recovery phase the ratio of the amino acids of aspartate family increased (DETA/NO: by $13 \%$; l-NNA: by 26\%) and that of the amino acids of glutamate family decreased (DETA/NO: by 12\%, l-NNA: $15 \%$ ) after the modification of NO levels, and this effect was strengthened (additional $23 \%$ and $21 \%$ increase) by the simultaneous addition of $\mathrm{NaCl}$ in the case of aspartate family.

The quantity of amino acids increased in the treatments, but generally decreased to the control level during the recovery phase (Figs. 5-9). Four amino acids of the glutamate family were present in detectable quantities (Fig. 5). The amount of glutamate increased (to $151-217 \%$ ) in all treatments after 3 days, but subsequently (11 days treatment) decreased to the control or lower levels in the case of $\mathrm{NaCl}$ (to 59\%), DETA $+\mathrm{NaCl}$ (to $75 \%$ ), l-NNA $+\mathrm{NaCl}$ (to 80\%) and $\mathrm{KNO}_{2}$ (to 105\%) (Fig. 5A). The glutamine concentration exhibited a great increase after 11-day $\mathrm{NaCl}$ (7.6-fold), l-NNA (6- fold) and l-NNA + NaCl (5.3-fold) treatments 
(Fig. 5B). The greatest increase was observed for proline when the plants were treated with $\mathrm{NaCl}$ alone (35-fold) or in combination with other compounds $(\mathrm{NaCl}+$ DETA/NO: 58-fold, $\mathrm{NACl}+$ DETA: 13-fold, $\mathrm{NaCl}+$ l-NNA: 40-fold) for 11 days (Fig. 5C).

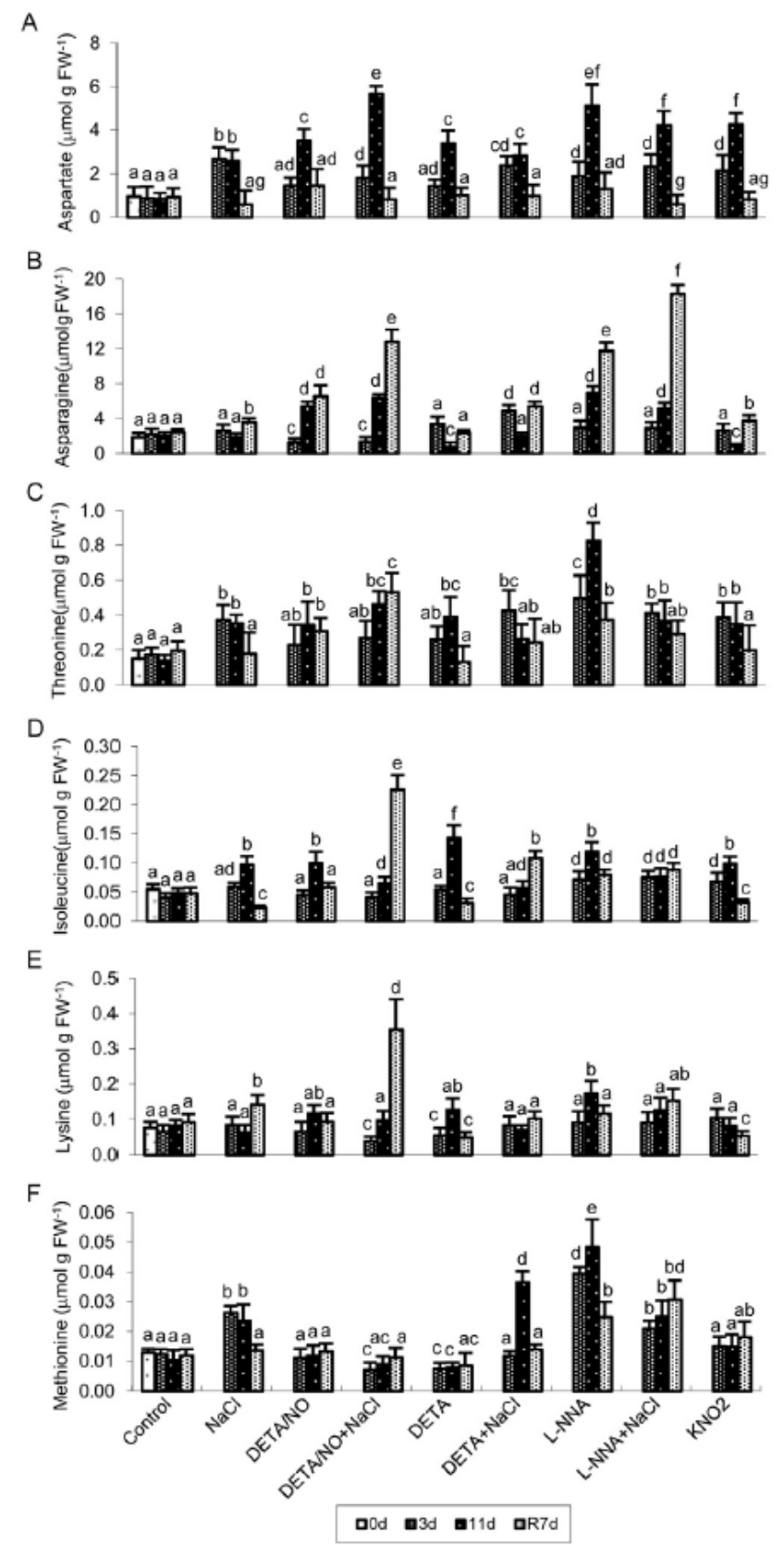

\begin{abstract}
Fig. 6. Effect of NO on salt-induced changes in the concentration of amino acids belonging to the aspartate family. The amounts of aspartate (A; SD: 0.17 ), asparagine (B; SD: 0.28), threonine (C; SD: 0.017), isoleucine (D; SD: 0.012), lysine (E; SD: 0.015 ) and methionine (F; SD: 0.002 ) were measured in the youngest fully developed leaves after 0,3 and 11 days of treatment with various compounds and after 7 days of recovery (without these compounds). $\mathrm{NaCl}: 150 \mathrm{mM}$; DETA/NO (NO-donor): $5 \mu \mathrm{mol}$; DETA: $5 \mu \mathrm{mol}$; L-NNA (inhibitor of NO-synthesis): $1 \mathrm{mM} ; \mathrm{KNO}_{2}$ (precursor of NO): $150 \mathrm{mM}$. The values indicated by different letters are significantly different at $p \leq 0.05$ level.
\end{abstract}

At the end of the recovery phase, as in the case of Gln, its level was much greater (5.5-15-fold) after the addition of DETA/ $\mathrm{NO}+\mathrm{NaCl}$ than in the other treatments. The -y-aminobutyrate (GABA) concentration was increased (2.6-4-fold) after 11 days by all the treatments, but decreased (by 60-92\%) during the recovery phase except for $\mathrm{NaCl}$ (Fig. 5D). The greatest increase was detected when l-NNA was applied alone (5.7-fold) or with $\mathrm{NaCl}$ (6.4-fold). Interestingly, arginine could be 
detected only in very low concentrations (0.05-0.1 $\mu \mathrm{mol} \mathrm{g} \mathrm{FW}^{-1}$ ) after 3 days in plants treated with $\mathrm{NaCl}$ alone or combined with other compounds (data not shown) which can be explained by its low basic level and by the greatly increased use of its precursor, Glu for Pro and Gln synthesis.

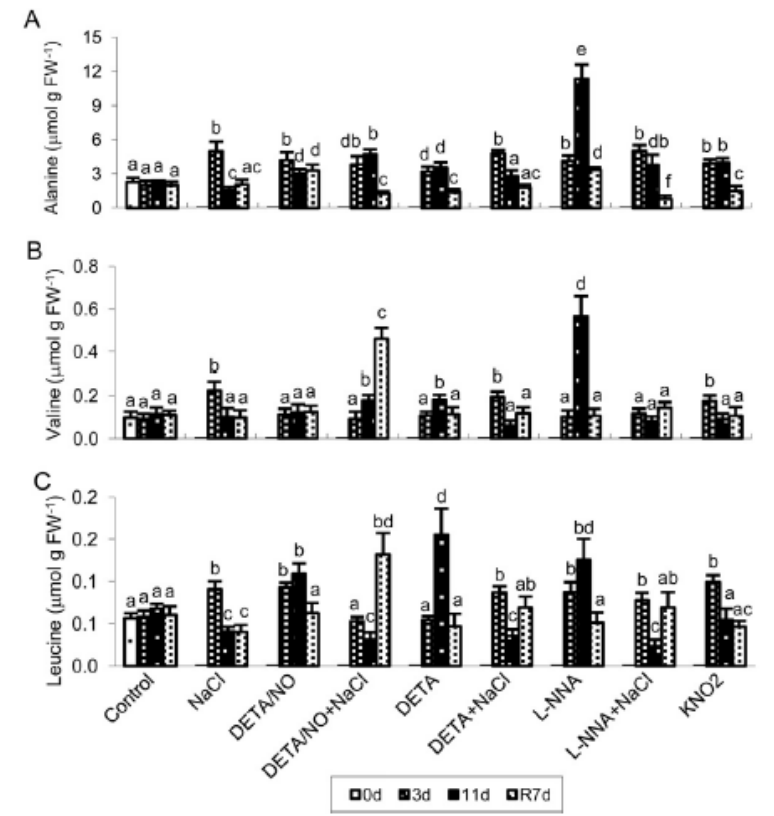

Fig. 7. Effect of NO on salt-induced changes in the concentration of amino acids belonging to the pyruvate family. The amounts of alanine (A; SD: 0.38 ), valine (B; SD: 0.015 ) and leucine (C; SD: 0.013 ) were measured in the youngest fully developed leaves after 0,3 and 11 days of treatment with various compounds and after 7 days of recovery (without these compounds) $\mathrm{NaCl}: 150 \mathrm{mM}$ : DETA/NO (NO-donor): 5 . of $\mathrm{NO}$ ): $150 \mathrm{mM}$. The values indicated by different letters are significantly different at $p \leq 0.05$ level.

Six amino acids of the aspartate family could be detected. All the chemicals induced a large increase (3-6.7-fold) in the aspartate content after 11 days, the greatest effect being detected after treatment with DETA/NO + $\mathrm{NaCl}$ (6.7-fold) and l-NNA (6-fold) (Fig. 6A). In contrast to Asp, the asparagine content further increased during recovery (Fig. 6B). Its highest concentration was observed after the addition of DETA/NO $+\mathrm{NaCl}, 1-\mathrm{NNA}$ and l-NNA $+\mathrm{NaCl}$ (5.2-, 4.8- and 7.6-fold greater) compared to the control. The threonine content was increased (2.5-5.9fold) by all the compounds, and this increase was especially large (5.9-fold) at the end of the l-NNA treatment (Fig. 6C). Although the isoleucine and lysine contents were increased by almost all the compounds, their levels were at least 2 times greater after DETA/ $\mathrm{NO}+\mathrm{NaCl}$ addition than in the other treatments at the end of the recovery (Fig. 6D and E). The inhibition of NO synthesis resulted in a very large increase (4.6-fold) in the methionine concentration after 11 days (Fig. 6F).

The investigation of the pyruvate family revealed that both the alanine and valine contents were increased 5 -fold by l-NNA by the end of the 11-day treatment, while the other treatments had no or much weaker effect (Ala: max. 2.2-fold, Val: max. 1.6fold increase) (Fig. 7A and B). Much higher Val (4.2-fold) and Leu levels (2.2fold) were found after addition of DETA/ $\mathrm{NO}+\mathrm{NaCl}$ compared to the other treatments at the end of the one-week recovery phase (Fig. $7 \mathrm{~B}$ and $\mathrm{C}$ ). 
A

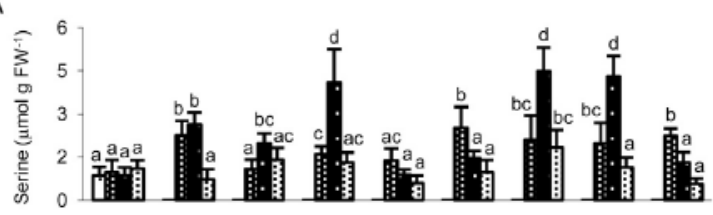

B

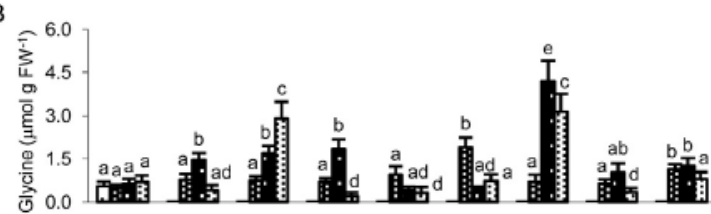

$\mathrm{C}$

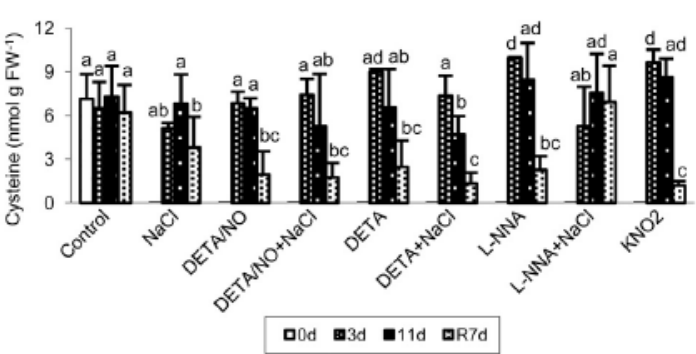

Fig. 8. Effect of NO on salt-induced changes in the concentration of amino acids belonging to the serine family. The amounts of serine (A; SD: 0.11 ), glycine (B; SD: 0.09 ) and cysteine (C; SD: 0.61$)$ were measured in the youngest fully developed leaves after 0,3 and 11 days of treatment with various compounds and after 7 days of recovery (without these compounds). $\mathrm{NaCl}: 150 \mathrm{mM}$; DETA/NO (NO-donor): 5 umol: DETA: 5 mol; L-NNA (inhibitor of NO-synthesis): $1 \mathrm{mM}$ : $\mathrm{KNO}$ (precursor at $p \leq 0.05$ level.

In the serine family, the serine and glycine concentrations were increased (1.5-5.2fold and 1.7-6.8-fold) and the cysteine content decreased (by 28-36\%) by most of the treatments (Fig. 8). The accumulation of Ser (5.2-fold) and Gly (6.8-fold) was induced by 11- day l-NNA treatment (Fig. 8A and B). In addition, a great increase in Ser content was detected after DETA/NO + NaCl (4.8-fold) and l-NNA + $\mathrm{NaCl}$ treatments (5-fold) and the Gly concentration was elevated (4.5-fold) in the DETA/NO-treated plants during the recovery.
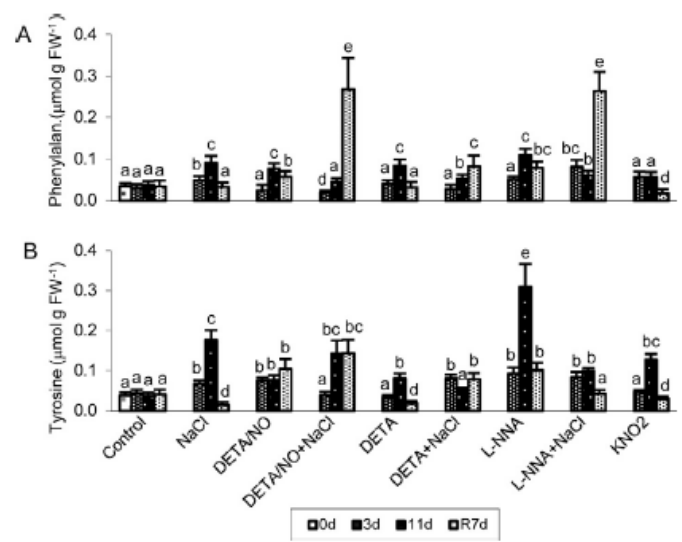

Fig. 9. Effect of NO on salt-induced changes in the concentration of amino acids belonging to the aromatic amino acid family. The amount of phenylalanine (A; SD: 0.012) and tyrosine (B. SD: 0.015) were measured in the youngest fully develop .

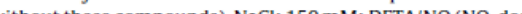
days of recovery (Without the compounds). NaCl. $500 \mathrm{mM}$; DETA/NO (NO-donor): $5 \mu \mathrm{mol}$; DETA. $5 \mu \mathrm{mol}$, L-NNA (inhibitor of NO-synthesis): $1 \mathrm{mM}$, $\mathrm{KNO}_{2}$ (precurso of $\mathrm{NO}$ ): $150 \mathrm{mM}$. The values indicated by different letters are significantly differen at $p \leq 0.05$ level. 


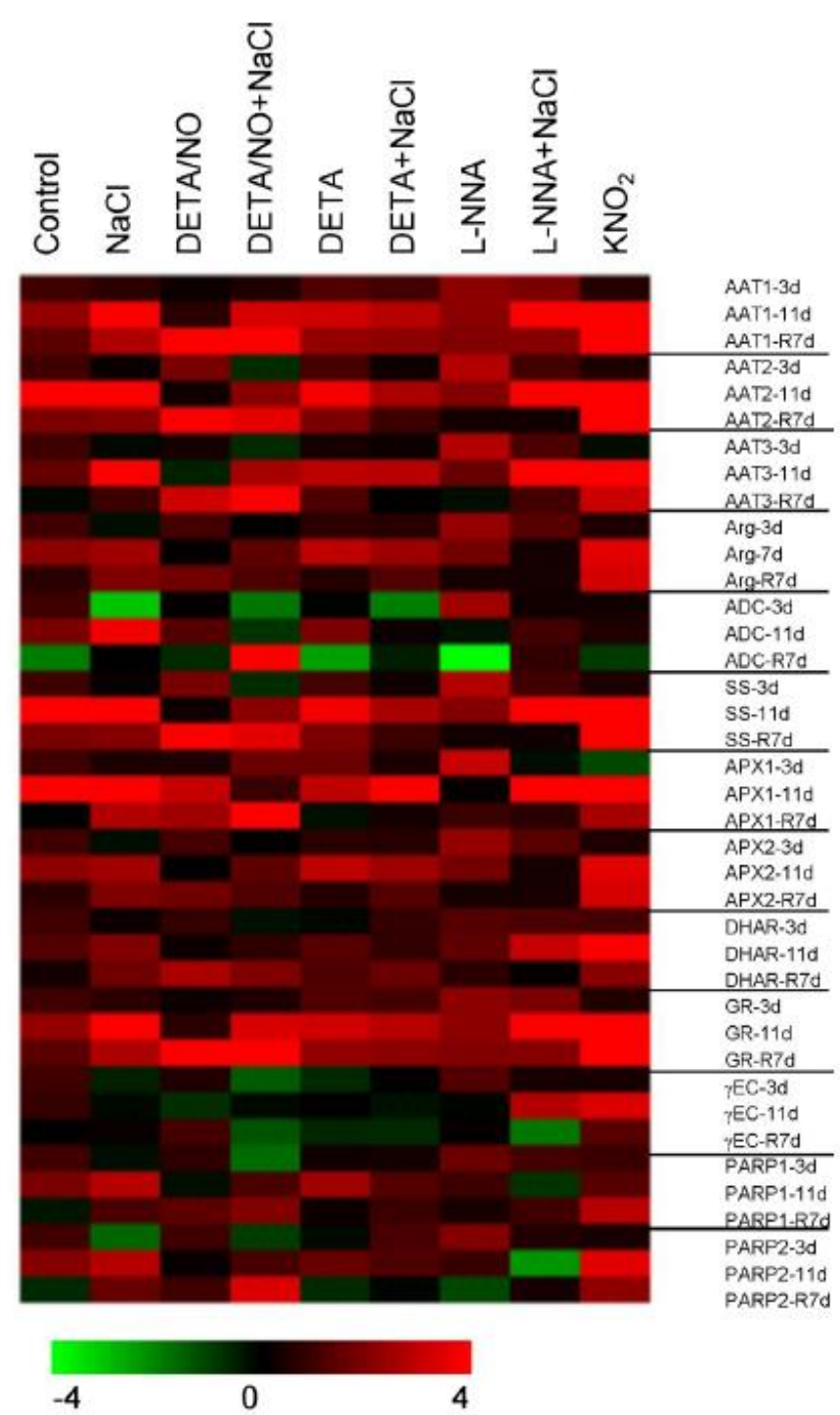

Fig. 10. Effect of NO on salt-induced changes in the expression of genes related to the amino acid metabolism, antioxidants and death processes. Expression was measured in the youngest fully developed leaves after 0,3 and 11 days of treatment with various compounds and after 7 days of recovery (without these compounds). All the values are given relative to the transcript levels measured before the treatments. Glyceraldehyde-3-phosphate dehydrogenase was used as a reference gene. $\mathrm{NaCl}$ : $150 \mathrm{mM}$; DETA/NO (NO-donor): $5 \mu \mathrm{mol}$; DETA: $5 \mu \mathrm{mol}$; L-NNA (inhibitor of NOsynthesis): $1 \mathrm{mM}$; $\mathrm{KNO}_{2}$ (precursor of $\mathrm{NO}$ ): $150 \mathrm{mM}$.

The Cys content was much lower (by 39-81\%) in the recovery phase after the treatments than in the control, except for l-NNA $+\mathrm{NaCl}$ addition (no change) (Fig. $8 \mathrm{C})$.

Among the members of the aromatic amino acid family, a 7.7- fold increase in the phenylalanine content was shown in plants treated with DETA/NO $+\mathrm{NaCl}$ and l$\mathrm{NNA}+\mathrm{NaCl}$ at the end of the one-week recovery period (Fig. 9A). 1-NNA induced a huge increase (8.3-fold) in the tyrosine content after 11 days, but this effect was much smaller (2.7-fold increase) when it was combined with $\mathrm{NaCl}$ (Fig. 9B). The other treatments increased (1.4-4.7-fold) the Tyr content, too.

\section{Gene expression studies}

Gene expression was investigated for several genes related to the amino acid, polyamine and antioxidant metabolism and to death processes. The transcript 
level of the three aspartate aminotransferase and arginase genes was increased (1.5-7.5-fold) by most of the treatments, except for DETA/NO after 11 days $\mathrm{NaCl}$ treatment (Fig. 10). The expression of arginase was induced by $\mathrm{NaCl}$ (2.5-fold), DETA (2.9-fold) and $\mathrm{KNO}_{2}$ (3.5-fold) after 11 days, and that of spermine synthase by DETA/NO (4.1-fold), l-NNA (5.3-fold) and $\mathrm{KNO}_{2}$ (7.5-fold) during recovery. The gene encoding APX1 was greatly induced (3-6-fold) by the various treatments after 11 days except DETA/NO $+\mathrm{NaCl}$ and l-NNA. The transcription of the GR gene was increased (2.2-7.5-fold, except for DETA/NO) and that of )'EC synthetase was not changed (except for l-NAA $+\mathrm{NaCl}-2.7$-fold increase and $\mathrm{KNO}_{2}-3.4$-fold increase) by most of the treatments. A great increase (2.8-fold) in transcript level of poly(ADP-ribose) polymerase1 (PARP1) and PARP2 was observed after $\mathrm{NaCl}$ treatment, while no change was detected after the DETA/NO $+\mathrm{NaCl}$ treatment.

\section{Discussion}

DETA/NO was successfully used to elevate the endogenous NO level and reduce salt-induced damage in maize (Keyster et al., 2012). This effect could be reversed by NO metabolic scavengers and inhibitors (Bai et al., 2011). In the present study, the protective role of NO against salt stress was confirmed in maize using DETA/NO and by inhibiting its synthesis with l-NNA. The improvement in salt tolerance was indicated not only by the growth data, but it was also shown at the gene expression level since the transcript level of PARP1 and PARP2, enzymes involved in the cell death process, was only increased by $\mathrm{NaCl}$ but not by DETA/NO $+\mathrm{NaCl}$ treatment in maize leaves. The reduction of salt-induced injuries by NO was confirmed by the simultaneous decrease in cell death and caspase-like activity in maize roots, too (Keyster et al., 2012).

Change in the GSH- and GSSG-dependent redox potential was suggested to be a marker of the stress-induced damages (Kranner et al., 2006). $\mathrm{NaCl}$ had a great effect on the redox environment in the maize leaves as it was shown by the increase in the amount of GSSG and in the redox potential of the GSH/GSSG redox pair. This influence of $\mathrm{NaCl}$ was modified by DETA/NO and l-NNA. The effect of NO on GSH synthesis was shown in Medicago trunculata, too (Innocenti et al., 2007). The protective effect of NO on tolerance to osmotic stress was mediated by GSH in Agropyron cristatum (Shan et al., 2012). NO-dependent changes in the amount and redox potential of glutathione may protect plants from stress-induced injuries directly by the removal of the excess of reactive oxygen species and indirectly by activation of defence mechanisms and adjustment of metabolism to the altered environmental conditions (Noctor et al., 2012). In addition, the role of the other components of redox system in the mediation of the effect of NO on the response to salt stress is indicated by the increased expression of GR and APX in the leaves of maize treated with DETA/NO $+\mathrm{NaCl}$ in the present study. Similarly, the combined application of DETA/NO and $\mathrm{NaCl}$ increased the activity of these two enzymes, and also that of GPX and DHAR in the roots of maize (Keyster et al., 2012). These results were confirmed in salt-stressed rice, where NO pre-treatment increased the activity of APX, GR, catalase and SOD and also the expression of stress-related genes (Uchida et al., 2002). The activation of antioxidants by NO during salt stress reduces 
injuries as it was shown in chickpea and soybean (Sheokand et al., 2008; Simaei et al., 2011).

Salt-induced changes in the amino acid metabolism were affected by NO in maize as shown by the alterations in the amino acid composition and in the amount of several amino acids. The effect of $\mathrm{NO}_{2}{ }^{-}$as an $\mathrm{NO}$ donor in plants was also tested (Crawford and Guo, 2005), and it was found to have an effect very similar to that of DETA/NO on the time-course of changes in the concentrations of several amino acids (Gln, Pro, GABA, Ile, Val, Cys).

Although NO affected the salt-induced accumulation of almost all the amino acids, its greatest effect was observed in the case of Pro, which effectively reduces $\mathrm{NaCl}$-induced damage as an osmoprotectant (Simon-Sarkadi et al., 2007). The Pro content in DETA/NO-treated plants was several times higher than that in the other treatments both after 3 days stress and during recovery. Similarly to the present findings, NO affected Pro concentration during salt stress in cabbage (López-Carrion et al., 2008). The NO-induced Pro accumulation was a result of enhanced Pro synthesis and decreased degradation in maize (Yang and Gong, 2009). Although a relationship between the NO and the Pro precursor Glu was found in tobacco cells, where the involvement of a Glu-receptor in NO production and plant defence signalling was observed (Vatsa et al., 2011), the pattern of saltand NO-dependent changes for Pro and Glu was not similar in maize in the present experiment. This difference can be explained by the possible simultaneous use of Glu for Pro, GSH and/or polyamine synthesis. Both GSH and polyamines have important role in the reduction of injuries occurring during abiotic stress (SimonSarkadi et al., 2007; Kello" s et al., 2008). The increased need for Glu because of greater Pro, GSH and polyamine synthesis may be ensured from the enhanced catabolism of Lys described in stressed plants (Galili et al., 2001). However, NO did not activate Lys catabolism in the present experimental system, since greater accumulation of Lys was observed in maize seedlings treated with DETA/NO $+\mathrm{NaCl}$ compared to $\mathrm{NaCl}$-treated ones during the recovery.

Besides Pro, the branched-chain amino acids, Ile, Val and Leu, accumulated to a much greater extent during the recovery phase after the addition of DETA/NO + $\mathrm{NaCl}$ than after the other treatments, indicating their involvement in the NOmediated response to salt stress. In the case of Ile this change may be the result of a simultaneous increase in the level of its precursor Thr after the DETA/NO + $\mathrm{NaCl}$ treatment. A recent study emphasized the importance of branched-chain amino acids as osmolytes which function might contribute to the improved salt tolerance in the present experiment (Joshi et al., 2010).

The inhibition of NO synthesis increased the Gln and GABA levels, but this effect was similar in both the absence and presence of $\mathrm{NaCl}$, therefore cannot be related to a protective mechanism in maize. However, the results obtained in tobacco (Akc, ay et al., 2012) suggest that the increase in the GABA content induced by inhibition of NO synthesis using l-NNA may have a protective role during salt stress. NO and GABA regulate mutually the concentration of each other, since GABA also affected 
NO synthesis in potato (Floryszak-Wieczorek et al., 2012). Similarly to maize, the effect of NO on Gln could be shown in root nodules of Medicago trunculata, where a Gln synthase was found to be a target of NO (Melo et al., 2011). In contrast to Gln and GABA, l-NNA only increased the concentrations of Thr, Ala and Tyr to very high levels compared to the other treatments in the absence of $\mathrm{NaCl}$, indicating that NO has a negative regulatory role on the concentration of these three amino acids. Interestingly, the Gly concentrations reached high levels after both the addition of NO donor and the inhibition of NO synthesis. This effect disappeared in the presence of $\mathrm{NaCl}$. Compared to Gly the opposite changes were observed for Phe, the level of which was high after the combined application of $\mathrm{NaCl}$ and DETA/NO or l-NNA, but low if these compounds were added without salt. Thus, the Phe level was only affected by NO during salt stress. Interestingly, higher and lower NO levels had a similar effect on the Gly and Phe metabolism which contradiction may be explained by possible induction of their synthesis by high NO levels and by possible inhibition of their degradation at low NO concentration.

In conclusion, modification of NO levels affected salt-induced, glutathionedependent redox changes and simultaneously the level of several amino acids. These could be independent effects, but it cannot be excluded that the influence of NO on free amino acid concentration is mediated by redox signalling. The observed much higher Pro content in plants treated with both $\mathrm{NaCl}$ and DETA/NO during recovery, may contribute to the protective effect of NO against salt stress.

\section{Acknowledgements}

This work was supported by a joint grant from the EU, the European Social Fund and the National Development Agency (TÁMOP-4.2.2/B-10/1-2010-0025, University of Pannonia), and by the Hungarian Scientific Research Fund (OTKA K 83642, CNK 80781). 


\section{References}

Abd El-Samad HM, Shaddad MAK, Barakat N. Improvement of plants salt tolerance by exogenous application of amino acids. J Med Plants Res 2011;5: 5692-9.

Akc, ay N, Bor M, Karabudak T, Ozdemir F, Türkan I. Contribution of gamma amino butyric acid (GABA) to salt stress responses of Nicotiana sylvestris CMSII mutant and wild type plants. J Plant Physiol 2012;169:452-8.

Alcázar R, Marco F, Cueves JC, Patron M, Ferrando A, Carrasco P, et al. Involvement of polyamines in plant response to abiotic stress. Biotechnol Lett 2006;28:1867-76.

Altpeter F, Varshney A, Abderhalden O, Douchkov D, Sautter C, Kumlehn J, et al. Stable expression of a defence-related gene in wheat epidermis under transcriptional control of a novel promoter confers pathogen resistance. Plant Mol Biol 2005;57:271-83.

Bai X, Yang L, Yang Y, Ahmad P, Yang Y, Hu X. Deciphering the protective role of nitric oxide against salt stress at the physiological and proteomic levels in maize. J Proteome Res 2011;10:4349-64.

Crawford NM, Guo F-Q. New insights into nitric oxide metabolism and regulatory functions. Trends Plant Sci 2005;10:195-200.

Ding M, Hou P, Shen X, Wang M, Deng S, Sun J, et al. Salt-induced expression of genes related to $\mathrm{Na}(+) / \mathrm{K}(+)$ and ROS homeostasis in leaves of saltresistant and salt-sensitive poplar species. Plant Mol Biol 2010;73:25169.

El-Shabrawi H, Kumar B, Kaul T, Reddy MK, Singla-Pareek SL, Sopory SK. Redox homeostasis, antioxidant defense, and methylglyoxal detoxification as markers for salt tolerance in Pokkali rice. Protoplasma 2010;245:85-96.

Fan HF, Du CX, Guo SR. Effect of nitric oxide on proline metabolism in cucumber seedlings under salinity stress. J Am Soc Hortic Sci 2012;137:127-33.

Floryszak-Wieczorek J, Arasimowicz-Jelonek M, Milczarek G, Janus L, PawlakSprada S, Abramowski D, et al. Nitric oxide-mediated stress imprint in potato as an effect of exposure to a priming agent. Mol Plant Microbe Interact 2012;25: 1469-77.

Foyer $\mathrm{CH}$, Noctor G. Ascorbate and glutathione: the heart of the redox hub. Plant Physiol 2011;155:2-18.

Galili G, Tang GL, Zhu XH, Gakiere B. Lysine catabolism: a stress and development super-regulated metabolic pathway. Curr Opin Plant Biol 2001;4:261-6.

Gémes K, Poór P, Horváth E, Kolbert Z, Szopkó D, Szepesi Á, et al. Cross-talk between salicylic acid and $\mathrm{NaCl}$-generated reactive oxygen species and nitric oxide in tomato during acclimation to high salinity. Physiol Plant 2011;142: 179-92.

Innocenti G, Pucciariello C, Le Gleuher M, Hopkins J, de Stefano M, Delledonne $\mathrm{M}$, et al. Glutathione synthesis is regulated by nitric oxide in Medicago truncatula roots. Planta 2007;225:1597-602. 
Joshi V, Joung JG, Fei Z, Jander G. Interdependence of threonine, methionine and isoleucine metabolism in plants: accumulation and transcriptional regulation under abiotic stress. Amino Acids 2010;39:933-47.

Kello" s T, Tímár I, Szilágyi V, Szalai G, Galiba G, Kocsy G. Stress hormones and abiotic stresses have different effects on antioxidants in maize lines with different sensitivity. Plant Biol 2008;10:563-72.

Keyster M, Klein A, Ludidi N. Caspase-like enzymatic activity and the ascorbate-glutathione cycle participate in salt stress tolerance of maize conferred by exogenously applied nitric oxide. Plant Signal Behav 2012;7: 349-6o.

Kocsy G, Athmer B, Perovic D, Himmelbach A, Szü cs A, Vashegyi I, et al. Regulation of gene expression by chromosome 5A during cold hardening in wheat. Mol Gen Genomics 2010;283:351-63.

Kocsy G, von Ballmoos P, Rüegsegger A, Szalai G, Galiba G, Brunold C. Increasing the glutathione content in a chilling-sensitive maize genotype using safeners increased protection against chilling-induced injury. Plant Physiol 2001;127:1147-56.

Kranner I, Birtic S, Anderson KM, Pritchard HW. Glutathione half-cell reduction potential: a universal stress marker and modulator of programmed cell death? Free Radic Biol Med 2006;40:2155-65.

Kranner I, Grill D. Determination of glutathione and glutathione disulphide in lichens: a comparison of frequently used methods. Phytochem Anal 1996;7:24-8.

López-Carrion AI, Castellano R, Rosales MA, Ruiz JM, Romero L. Role of nitric oxide under saline stress: implications on proline metabolism. Biol Plant 2008;52:587-91.

Melo PM, Silva LS, Ribeiro I, Seabra AR, Carvalho HG. Glutamine synthetase is a molecular target of nitric oxide in root nodules of Medicago truncatula and is regulated by tyrosine nitration. Plant Physiol 2011;157:1505-17.

Menezes-Benavente L, Kernodle SP, Margis-Pinheiro M, Scandalios JG. Saltinduced antioxidant metabolism defenses in maize (Zea mays L.) seedlings. Redox Rep 2004;9:29-36.

Mhadhbi H, Fotopoulos V, Mylona PV, Jebara M, Elarbi Aouani M, Polidoros AN. Antioxidant gene-enzyme responses in Medicago truncatula genotypes with different degree of sensitivity to salinity. Physiol Plant 2011;141:201-14.

Moreau M, Lindermayr C, Durner J, Klessig DF. NO synthesis and signalling in plants - where do we stand? Physiol Plant 2010;138:372-83.

Noctor G, Mhamdi A, Chaouch S, Han Y, Neukermans J, Marquez-Garcia B, et al. Glutathione in plants: an integrated overview. Plant Cell Environ 2012;35:454-84.

Rai VK. Role of amino acids in plant responses to stresses. Biol Plant 2002;45:481-7. Schafer FO, Buettner GR. Redox environment of the 
cell as viewed through the redox state of the glutathione disulphide/glutathione couple. Free Radic Biol Med 2001;30:1191-212.

Sekmen AH, Türkan I, Takio S. Differential responses of antioxidative enzymes and lipid peroxidation to salt stress in salt-tolerant Plantago maritima and salt-sensitive Plantago media. Physiol Plant 2007;131:399-411.

Shan C, He F, Xu G, Han R, Liang Z. Nitric oxide is involved in the regulation of ascorbate and glutathione metabolism in Agropyron cristatum leaves under water stress. Biol Plant 2012;56:187-91.

Sheokand S, Kumari A, Sawhney V. Effect of nitric oxide and putrescine on antioxidative responses under $\mathrm{NaCl}$ stress in chickpea plants. Physiol Mol Biol Plants 2008;14:355-62.

Simaei M, Khavari-Nejad RA, Saadatmand S, Bernard F, Fahimi H. Effects of salicylic acid and nitric oxide on antioxidant capacity and proline accumulation in Glycine max L. treated with $\mathrm{NaCl}$ salinity. Afr J Agric Res 2011;6:3775-82.

Simon-Sarkadi L, Kocsy G, Sebestyén Z, Galiba G. Deletions of chromosome 5A affect free amino acid and polyamine levels in wheat subjected to salt stress. Environ Exp Bot 2007;60:193-201.

Stoeva N, Berova M, Vassilev A, Zlatev Z. Effect of exogenous polyamine diethylenetriamine on oxidative changes and photosynthesis in Astreated maize plants (Zea mays L.). J Cent Eur Agric 2005;3:367-74.

Uchida A, Jagendorf AT, Hibino T, Takabe T, Takabe T. Effects of hydrogen peroxide and nitric oxide on both salt and heat stress tolerance in rice. Plant Sci 2002;163:515-23.

Vaidyanathan H, Sivakumar P, Chakrabarty R, Thomas G. Scavenging of reactive oxygen species in $\mathrm{NaCl}$-stressed rice (Oryza sativa L.) differential response in salt-tolerant and sensitive varieties. Plant Sci 2003;165:1411-8.

Varga B, Janda T, László E, Veisz O. Influence of abiotic stresses on the antioxidant enzyme activity of cereals. Acta Physiol Plant 2012;34:84958.

Vatsa P, Chiltz A, Bourque S, Wendehenne D, Garcia-Brugger A, Pugin A. Involvement of putative glutamate receptors in plant defence signalling and NO production. Biochimie 2011;93:2095-101.

Wang H, Miyazaki S, Kawai K, Deyholos M, Galbraith DW, Bohnert HJ. Temporal progression of gene expression responses to salt shock in maize roots. Plant Mol Biol 2003;52:873-91.

Yang SL, Gong M. Effect of nitric oxide on proline accumulation and metabolic pathways in maize seedlings. J Plant Physiol Commun 2009;45:781-4.

Zhang YN, Wang LL, Liu YL, Zhang Q, Wei QP, Zhang WH. Nitric oxide enhances salt tolerance in maize seedlings through increasing activities of protonpump and $++\mathrm{Na} / \mathrm{H}$ antiport in the tonoplast. Planta 2006;224:54555.

Zhao LQ, Zhang F, Guo JK, Yang YL, Li BB, Zhang LX. Nitric oxide functions as a signal in salt resistance in the calluses from two ecotypes of reed. Plant Physiol 2004;134:849-57. 
Zheng CF, Jiang D, Liu FL, Dai TB, Liu WC, Jing Q, et al. Exogenous nitric oxide improves against mitochondrial oxidative damage induced by high salinity. Environ Exp Bot 2009;67:222-7. 